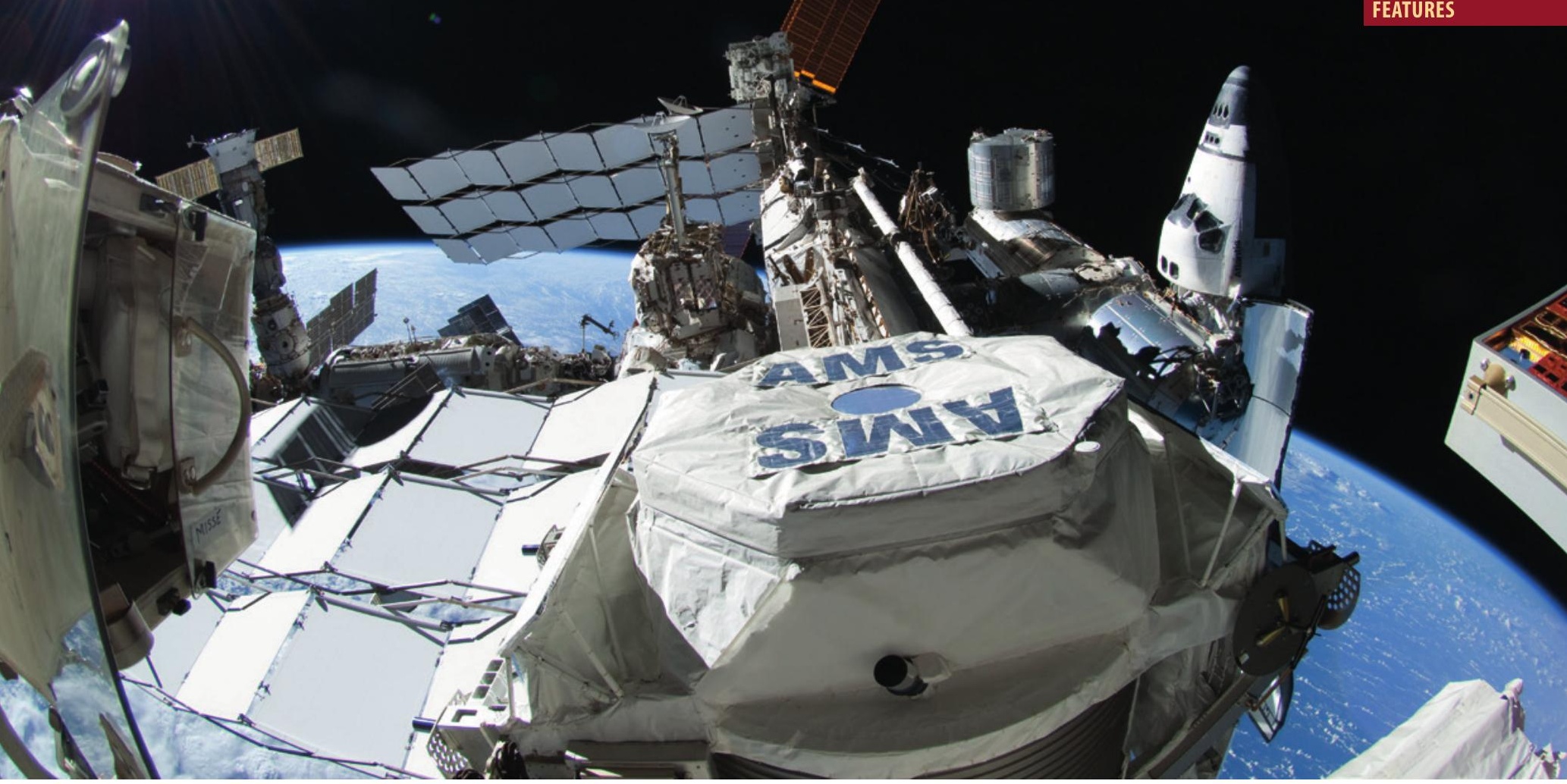

\title{
FIRST RESULT FROM
}

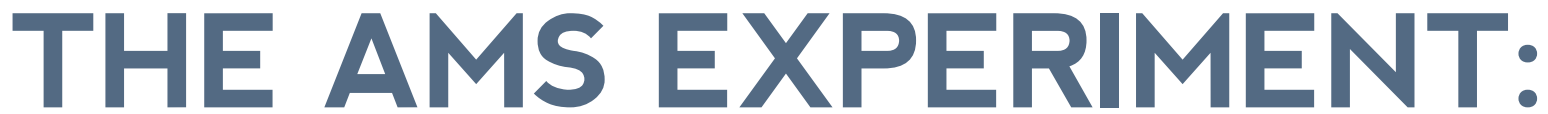

\section{THE BEGINNING OF A RICH COSMIC RAY PROGRAM}

ـ Martin Pohl - Center for Astroparticle Physics (CAP Genève) Université de Genève - DOI: 10.1051/epn/2014302

\section{More than 100 years after the discovery of cosmic rays by Victor Hess, our knowledge} of their sources and transport is still rather rudimentary. This does not only concern the astrophysical phenomena, which create and accelerate charged particles, but also the potential role of particle physics and cosmological mechanisms.

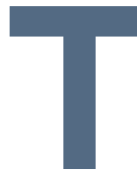

he Alpha Magnetic Spectrometer (AMS) [1] is a powerful and sensitive particle physics spectrometer for the observation of cosmic rays in space. As seen in Fig. 1, AMS is located on the exterior of the International Space Station (ISS). Since its installation on 19 May 2011 it has measured about 40 billion cosmic rays in the $\mathrm{GeV}$ to $\mathrm{TeV}$ energy range. Its permanent magnet and array of precision particle detectors collect and identify charged cosmic rays passing through. Over its long-duration mission on the ISS, AMS will record signals from 16 billion cosmic rays every year and transmit them to Earth for analysis by the AMS Collaboration. Beginning of April 2013, the Collaboration published its first physics result in Physical Review Letters [2]. Later this summer, new results were contributed to the $33^{\text {rd }}$ International Cosmic Ray Conference in Rio de Janeiro. These are the first of many physics results to be reported.
The first publications from the AMS Experiment are a major milestone for the AMS international collaboration [2]. Hundreds of scientists, engineers, technicians and students from all over the world have worked together for over 18 years to make AMS a reality. The collaboration represents 16 countries from Europe, Asia and North America (Finland, France, Germany, Italy, the Netherlands, Portugal, Spain, Switzerland, Romania, Russia, Turkey, China, Korea, Taiwan, Mexico and the United States) under the leadership of Nobel Laureate Samuel C.C. Ting of M.I.T. The collaboration continues to work closely with the NASA AMS Project Management team from Johnson Space Center as it has throughout the entire process. Many countries have made important contributions to the AMS detector construction and are presently participating in the data analysis. 
AMS was constructed at universities and research institutes around the world and assembled at the European Organization for Nuclear Research, CERN, Geneva, Switzerland. It was launched by NASA to the ISS as the primary payload onboard the final mission of space shuttle Endeavour (STS-134) on 16 May 2011. Once installed on 19 May 2011,AMS was powered up and immediately began collecting data from primary sources in space. Since June 2011, these are routinely transmitted to the AMS Payload Operations Control Center located at CERN, Geneva, Switzerland.

Once AMS became operational, the first task for the Collaboration was to ensure that all instruments and systems performed as designed and as tested on the ground. The AMS detector, with its multiple redundancies, has proven to perform flawlessly in space. Over more than two years in flight, AMS collaborators have gained invaluable operational experience in running a precision spectrometer in space and mitigating the hazardous conditions to which AMS is exposed as it orbits the Earth every 90 minutes. Conditions like this are not encountered by ground-based accelerator experiments or satellite-based experiments and require constant vigilance in order to avoid irreparable damage. They include the extreme thermal variations caused by illumination from the Sun and the re-positioning of ISS onboard radiators and solar arrays. In addition, the AMS operators regularly transmit software updates from the AMS Payload Operation and Control Center at CERN to the AMS computers in space in order to match the regular upgrades of the ISS software and hardware.

\section{Positron fraction measurement}

In the initial 18 months period of space operations, from 19 May 2011 to 10 December 2012,AMS analyzed 25 billion primary cosmic ray events. Of these, an unprecedented number, 6.8 million, were unambiguously identified as electrons and their antimatter counterpart, positrons, in the energy range 0.5 to $350 \mathrm{GeV}$.

Electrons and positrons are identified by the accurate and redundant measurements provided by the various AMS instruments against a large background of protons. Positrons are clearly distinguished from this background through the robust rejection power of AMS of more than one in one million. The total number of positrons identified by AMS, in excess of 400000 , is the largest number of energetic antimatter particles directly measured and analyzed from space.

In its first publication [2], AMS presents the positron fraction (ratio of the positron flux to the combined flux of positrons and electrons) in the energy range 0.5 to $350 \mathrm{GeV}$. We have observed that from 0.5 to $10 \mathrm{GeV}$, the fraction decreases with increasing energy. The fraction then increases steadily between $10 \mathrm{GeV}$ and $250 \mathrm{GeV}$. Yet the slope (rate of growth) of the positron fraction decreases by an order of magnitude from 20 to $250 \mathrm{GeV}$. At energies above $250 \mathrm{GeV}$, the spectrum appears to flatten, but to study the behaviour above $250 \mathrm{GeV}$ requires more statistics - the data reported represent $\sim 10 \%$ of the total expected. The positron fraction spectrum exhibits neither structure nor time dependence. The positron-to-electron ratio shows no anisotropy [3] indicating the energetic positrons are not coming from a preferred direction in space. Together, these features show

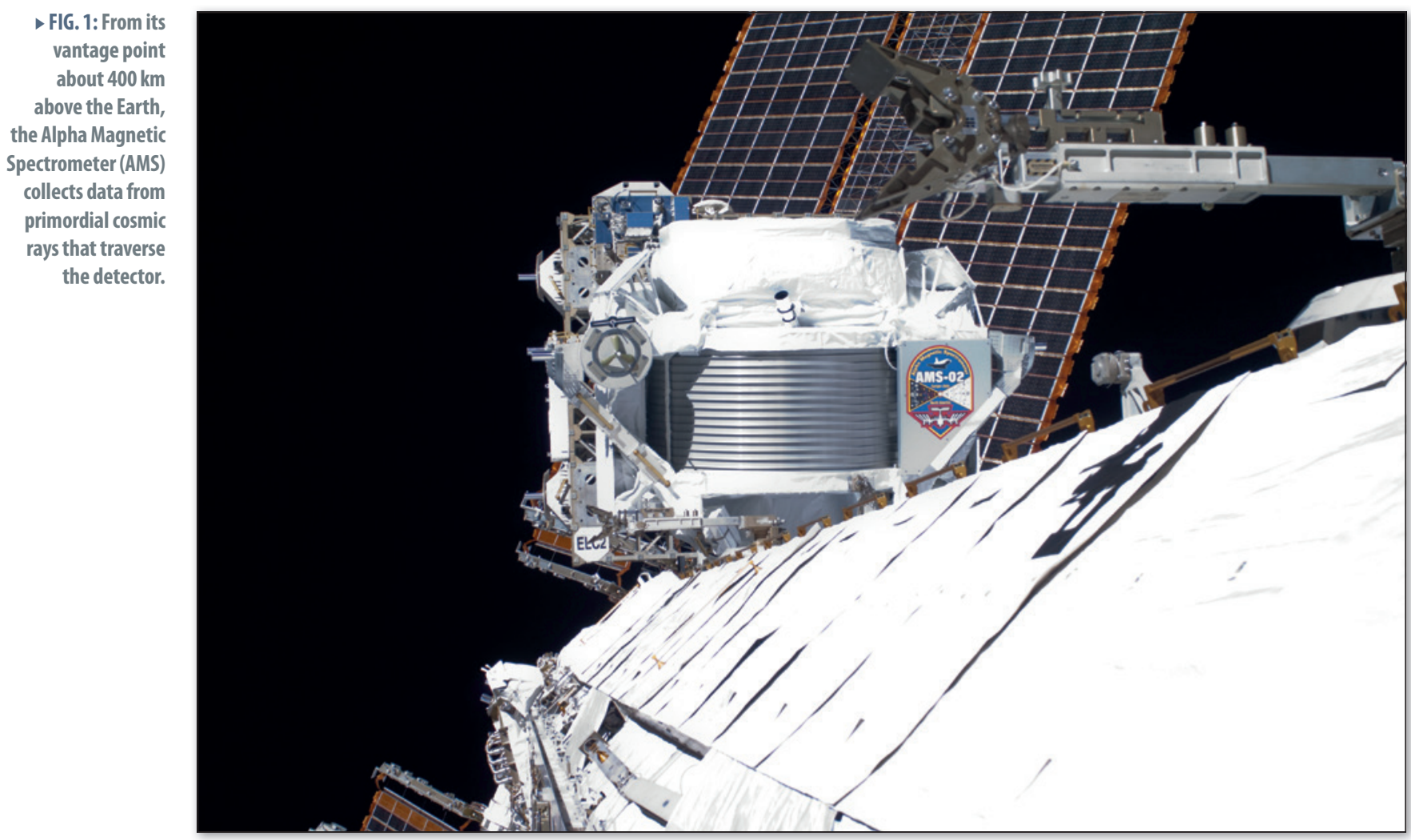


evidence of new physics phenomena. Fig. 2 illustrates the AMS data presented in the first publication. Even with the high statistics, 6.8 million events, and the accuracy of AMS, the fraction shows no fine structure.

The exact shape of the spectrum as shown in Fig. 2, extended to higher energies, will ultimately determine whether this spectrum originates from the collision of dark matter particles or from pulsars in the galaxy. The high level of accuracy of these data indicates that AMS may soon resolve this issue. In fact, latest results [4] confirm the finding that at high energies the positron fraction no longer increases.

Over the last few decades there has been much interest in the positron fraction [5] from primary cosmic rays by both particle physicists and astrophysicists. The underlying reason is that by measuring the ratio between positrons and electrons and by studying the bahaviour of any excess across the energy spectrum, a better understanding of the origin of dark matter and other physics phenomena may be obtained.

The first AMS result has been analyzed using several phenomenological models, one of which is described in the paper and included in Fig. 2. This generic model, with diffuse electron and positron components and a common source component, fits the AMS data surprisingly well. This agreement indicates that the positron fraction spectrum is consistent with electron and positron fluxes, each of which is the sum of a separate diffuse spectrum and a single energetic common source. In other words, a significant portion of the high-energy electrons and positrons originate from an as yet unidentified common source. This is indeed corroborated by a recent separate measurement of the absolute electron and positron fluxes [6], up to $500 \mathrm{GeV}$ and $350 \mathrm{GeV}$ respectively, as well as the sum of the two [7] up to $700 \mathrm{GeV}$. More specific models [8] based on dark matter self annihilation and/ or pulsar sources in the Milky Way have been published immediately after the release of the AMS data.

\section{Fluxes of cosmic nuclei}

Hydrogen and Helium nuclei represent the most abundant components of cosmic rays. Their absolute flux and spectral shape are fundamental data to understand their origin and propagation history in our galaxy and to study solar phenomena. Data preceding AMS measurements have indicated that there might be variations of the spectral shape above $100 \mathrm{GeV}$ [9]. Two years of data taking on the ISS permit to deliver a precision measurement of the proton [10] and Helium flux [11] for energies ranging from the geomagnetic cut-off up to several TeV. The directly measured kinematic quantity for each particle is its magnetic rigidity $p / Z$,i.e., its momentum per unit charge.

About $1 \%$ of cosmic rays are nuclei heavier than Helium. Their absolute fluxes and the ratio of primary nuclei to the secondary nuclei produced by spallation on the interstellar medium are important input to understand

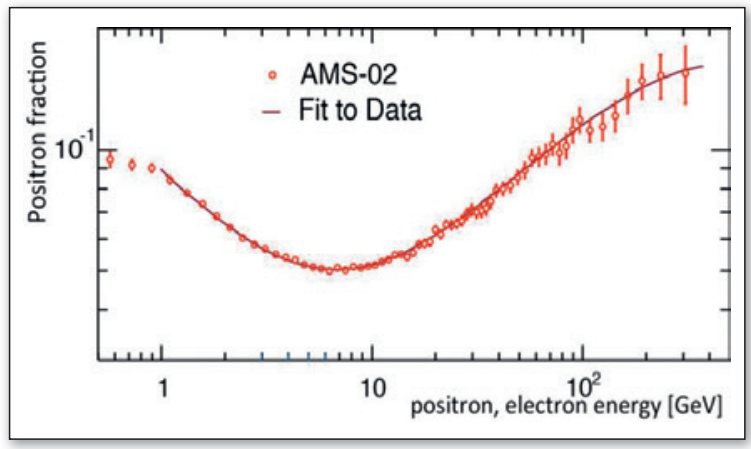

4 FIG. 2: The positron fraction measured by AMS demonstrates excellent agreement with the simple model described in the text. It shows no fine structure.

the acceleration and propagation mechanisms of cosmic rays in our galaxy. As a first step, AMS has presented results on the ratio of Boron to Carbon fluxes [12] between a few hundred $\mathrm{MeV}$ and a few hundred $\mathrm{GeV}$ of kinetic energy per nucleon.

To differentiate between nuclei, single track events are selected, with a charge measured accurately in the tracking detector. Redundancy in charge measurement by the time-of-flight system and tracking device determines the efficiency of charge determination from data alone; it is more than $99 \%$ over the full rigidity range. Cross-feed between the two species and background from heavier ions is much below the percent level. As an example, Fig. 3 shows the nuclear charge measured by the tracker for samples of proton and Helium candidates selected using the time-of-flight scintillators.

The absolute particle flux is obtained from the raw counting rate, dividing by the exposure time, the acceptance in surface and solid angle, the trigger and identification efficiencies, as well as the interval in energy or rigidity. The AMS results represent a big leap forward in terms of statistical and systematical accuracy. Both Hydrogen and Helium nuclei show a smooth power law spectrum without features or breaks in the accessible energy region. Likewise, the ratio of the spectra for Boron and Carbon nuclei is measured with unprecedented accuracy and found to decrease smoothly from the geomagnetic cut-off all the way to energies of $670 \mathrm{GeV}$ per nucleon.

The flux below $30 \mathrm{GV}$ is affected by solar activity. Because of its large acceptance, AMS can determine the proton flux every day with an accuracy of about $1 \%$.

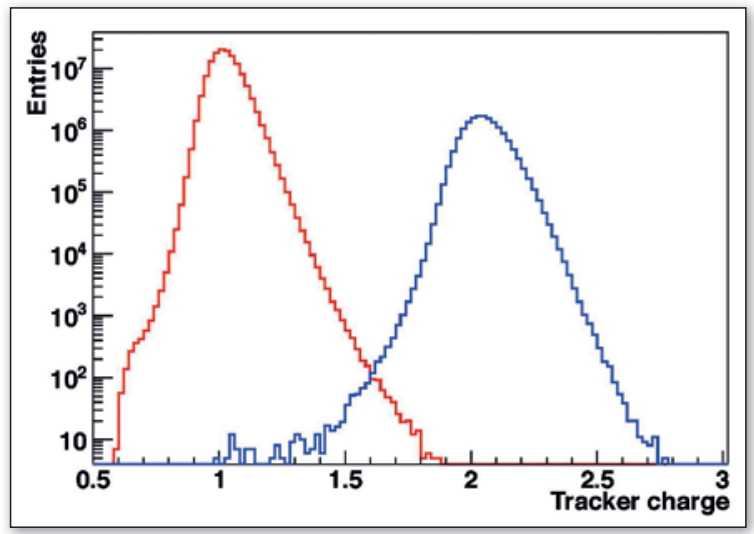

4 FIG. 3: Distribution of the charge measured by the tracking detector for samples of events identified as protons (red) or Helium (blue) by the time-offlight counters. 
Fig. 4 shows the time variation of the daily flux in magnetic rigidity intervals situated between 1 and $100 \mathrm{GV}$. The fluxes are normalized in the beginning of the period. A gradual decrease is observed for rigidities below about 10 GV due to solar modulation. The large spike on 7 March 2012 corresponds to an X5.4-class solar flare, which was the strongest eruption in 2012. We subsequent ly observed a large Forbush decrease of the flux up to 30 $\mathrm{GV}$, which lasted about three

weeks. A spike on 17 May 2012 corresponds to a M5.1 solar flare and the first Ground Level Enhancement event in Solar Cycle 24. A few other spikes corresponding to $\mathrm{X}$-class solar events were observed on 9 August 2011 and 27 January 2012.

\section{Outlook}

As interesting as these first results may be, they are only the tip of the iceberg. AMS is a magnetic spectrometer with the ability to explore new physics because of its precision, statistics, energy range, capability to identify different particles and nuclei as well as its long data-taking period in space.AMS is designed to measure the energy- and time-dependent fluxes of cosmic nuclei to an unprecedented degree of precision, to better understand the propagation, the confinement mechanisms of cosmic rays in the Galaxy and the strength of their interactions with interstellar media. Another high-priority research topic is the indirect search for dark-matter signals based on looking at the fluxes of particles such as electrons,

v FIG. 4: Daily variation of the normalized proton flux, for the rigidity ranges specified by the color code. AMS has observed a gradual decrease in the low rigidity region as well as spikes associated with solar events. that hundreds of billions of cosmic rays will be measured by AMS throughout the lifetime of the International Space Station. With the wealth of data collected, the Collaboration will also explore other topics such as the search for new physics and astrophysics phenomena like residual nuclear antimatter and strangelets. AMS will thus provide new, accurate information over the lifetime of the Space Station as the AMS detector continues its mission to explore new physics phenomena in the cosmos.

\section{About the author}

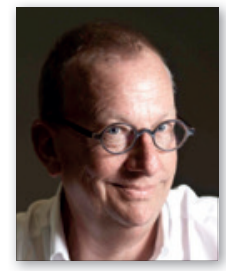

Martin Pohl obtained his $\mathrm{PhD}$ from RWTH Aachen in the 1970s. He has been working on experimental particle physics at the PETRA (DESY) and LEP (CERN) colliders before turning to astroparticle physics in space. Besides AMS, his projects include the POLAR X-ray polarimeter for the Chinese space laboratory Tiangong 2; the X-ray observatory LOFT, which is an ESA M-class mission candidate; and DAMPE, a high-energy particle detector for a free flying Chinese satellite. He is the director of the department for particle and nuclear physics (DPNC) at University of Geneva, and co-founder of the Center for Astroparticle Physics, CAP Genève.

\section{References}

[1] See: www.ams02.org

[2] AMS Collaboration, M. Aguilar et al., Phys. Rev. Lett. 110, 141102 (2013)

[3] See also http://press.web.cern.ch/tags/ams and M. Aguilar, CERN Courier, Sept. 27, 2013, http://cerncourier.com/cws/ article/cern/54675

[4] J. Casaus, "Determination of the positron anisotropy with AMS",

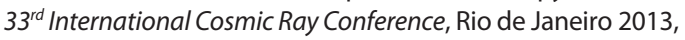
to be published in the proceedings.

[5] A. Kounine, "Precision measurement of the positron fraction in primary cosmic rays of $0.5-350 \mathrm{GeV}^{\prime}, 33^{\text {rd }}$ International Cosmic Ray Conference, Rio de Janeiro 2013, to be published in the proceedings.

[6] TS93: R. Golden et al., Astrophys. J. 457, L103 (1996). Wizard/CAPRICE: M. Boezio et al., Adv. Sp. Res. 27-4, 669 (2001). HEAT: J. J. Beatty et al., Phys. Rev. Lett. 93, 241102 (2004); M. A. DuVernois et al., Astrophys. J. 559, 296 (2001). AMS-01: M. Aguilar et al., Phys. Lett. B 646, 145 (2007). PAMELA: P. Picozza, Proc. of the $4^{\text {th }}$ International Conference on Particle and Fundamental Physics in Space, Geneva, 5-7 Nov. 2012, to be published; O. Adriani et al., Astropart. Phys. 34, 1 (2010); O. Adriani et al., Nature 458, 607 (2009). Fermi-LAT: M. Ackermann et al., Phys. Rev. Lett. 108, 011103 (2012).

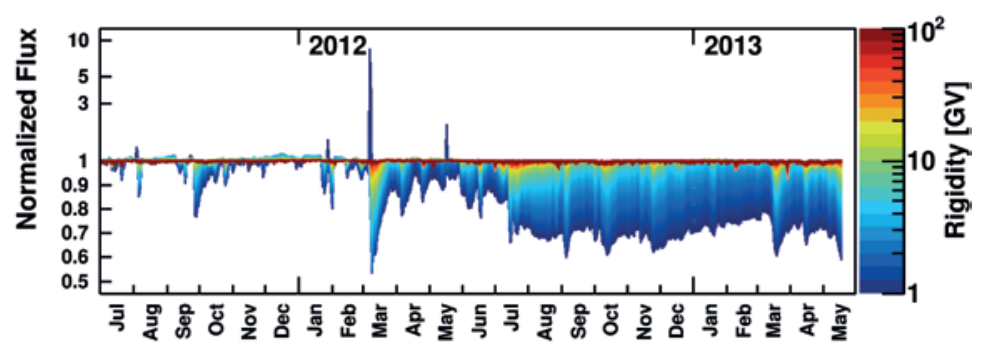

[7] S. Schael, "Precision measurement of the electron spectrum and the positron spectrum with AMS", $33^{r d}$ International Cosmic Ray Conference, Rio de Janeiro 2013, to be published in the proceedings.

[8] B. Bertucci, "Precision measurement of the e++e- spectrum

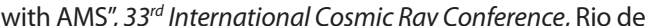
Janeiro 2013, to be published in the proceedings.

[9] See e.g. : Andrea De Simone, Antonio Riotto, Wei Xuec, CERNPH-TH/2013-054 (April 3, 2013); Tim Linden and Stefano Profumo, arXiv:1304.1791v1 [astro-ph.HE], (April 5, 2013); Peng-Fei Yin, Zhao-Huan Yu, Qiang Yuan and Xiao-Jun Bi, arXiv:1304.4128v1 [astro-ph.HE] (April 15, 2013)

[10] PAMELA collaboration, O. Adriani et al., Science 332, 69 (2011)

[11] S. Haino, "Precision measurement of the proton flux with AMS", $33^{\text {rd }}$ International Cosmic Ray Conference, Rio de Janeiro 2013 , to be published in the proceedings.

[12] V. Choutko, "Precision measurement of the cosmic ray Helium flux with AMS experiment", $33^{\text {rd }}$ International Cosmic Ray Conference, Rio de Janeiro 2013, to be published in the proceedings.

[13 ] A. Oliva, "Precision measurement of the cosmic ray Boron-to-Carbon ratio with AMS", $33^{\text {rd }}$ International Cosmic Ray Conference, Rio de Janeiro 2013, to be published in the proceedings. 